\title{
Estudio de satisfacción poblacional a la red vial intercomunitaria Quisapincha - Pasa
}

\section{Study of satisfactory population in intercommunity road between Quisapincha and Pasa}

\author{
María Elena Jerez Calero \\ Universidad de las Fuerzas Armadas-ESPE, Ecuador \\ Sonnia Lorena Llerena Cepeda \\ Universidad Técnica de Ambato, Ecuador \\ Ruth Zamora Sánchez \\ Universidad Técnica de Ambato, Ecuador
}

Autor para correspondencia: mejerez@espe.edu.ec, sonialllerena@uta.edu.ec,ra.zamora@uta.edu.ec Fecha de recepción: 12 de Octubre 2017 - Fecha de aceptación: 15 de Febrero de 2018

Resumen: El propósito del estudio fue examinar los cambios vividos por los pobladores de los sectores que conforman la vía intercomunitaria Quisapincha-Pasa, así como su nivel de satisfacción de la obra. Se tomó como referencia los cuestionarios desarrollados por el Instituto Nacional de Estadísticas y Censos (INEC), tanto el Censo de Población y Vivienda efectuado en 2010 como la Encuesta de Condiciones de Vida correspondiente al periodo 2013-2014; además, se definieron dos dimensiones: dimensión uno, Nivel de Satisfacción y Nivel de Insatisfacción; dimensión dos, Nivel Ni Satisfecho y Nivel Ni Insatisfecho. El cuestionario fue aplicado a una muestra de 401 personas. Los efectos de la obra pública recogidos mostraron que la construcción de la red vial intercomunitaria Quisapincha-Pasa ha contribuido a la accesibilidad, movilidad y acceso a servicios básicos por parte de los pobladores, así como una mejora en el tipo de vivienda, el acceso a distintos medios de transporte y de servicios básicos. Todo ello, lo recoge el índice de calidad de vida, que alcanzó un 12,49\%, esto es que existe una mejora en la calidad de vida de los pobladores, tomando como referencia el año 2010.

Palabras Clave: obra pública; calidad de vida; impacto socioeconómico; vías intercomunitarias

\begin{abstract}
The purpose of the study was to examine the changes experienced by the inhabitants of the sectors that make up the Quisapincha-Pasa intercommunity road, as well as their level of job satisfaction. Reference was made to the questionnaires developed by the National Institute of Statistics and Censuses (INEC), both the Population and Housing Census conducted in 2010 and the Living Conditions Survey for the period 2013-2014; In addition, two dimensions were defined: dimension one, Level of Satisfaction and Level of Dissatisfaction; Dimension two, Neither satisfied nor dissatisfied. The questionnaire was applied to a sample of 401 people. The effects of the public works collected showed that the construction of the intercommunity road network Quisapincha-Pasa has contributed to the accessibility, mobility and access to basic services by the inhabitants, as well as an improvement in the type of housing, access to Different means of transport and basic services. All of this is reflected in the quality of life index, which reached $12.49 \%$, that is, there is an improvement in the quality of life of the settlers, taking as reference the year 2010.

Key words: public work; quality of life; socio-economic impact; intercommunity roads
\end{abstract}




\section{Introducción}

Los caminos han sido siempre considerados como medios que facilitan la comunicación entre las localidades. Los factores que han contribuido a que este mejoramiento vial se produzca son el incremento del parque automotor y la necesidad de la población de movilizarse hacia los centros principales de abasto de bienes para el consumo.

Una infraestructura vial adecuada puede ser considerada como parte clave en el desarrollo económico de un país, debido a que sirve de enlace entre los centros de producción y consumo, generando comercio y estimulando el desplazamiento de la población de unas regiones a otras a lo largo del territorio nacional (Santos y Gomes, 2015). De igual manera, el acceso a los medios de transportes también está estructurado por la desigualdad social (Rydin et al., 2012).

Según el documento de Actualización del Plan de Desarrollo y Ordenamiento Territorial Tungurahua (GADM Ambato, 2015), la provincia dispone de una red de conectividad interna de primer nivel, conformada tanto por vías intercantonales como interparroquiales, las cuales unen las capitales cantonales y sus parroquias de manera directa con la capital provincial, la ciudad de Ambato, principal centro de abastecimiento de la zona centro del país.

No obstante, y a pesar de los avances conseguidos en la construcción y mejora de carreteras y caminos, aún existen territorios que no han logrado beneficiarse de esta dinámica territorial de desarrollo vial a pesar de estar muy cerca de la ciudad de Ambato. Un ejemplo de ello es la población que reside en la zona alta occidental de la provincia, ubicada a una cota superior a 2.800 metros sobre el nivel del mar. Este territorio se caracteriza por presentar elevados índices de pobreza (Villar y Ramírez, 2014), al igual que los pobladores de las localidades de Quisapincha y Pasa, con un $85,5 \%$ y un $93,7 \%$ respectivamente, los cuales son porcentajes muy altos de población quienes presentan necesidades básicas insatisfechas (INEC, 2010).

El panorama antes mencionado ha animado a las investigadoras a explorar cómo ha contribuido la construcción de la vía intercomunitaria Quisapincha-Pasa, analizando la percepción de los pobladores antes y después de la implementación de la obra. En concreto, el estudio se centra en conocer las diferencias percibidas por los habitantes de las parroquias Quisapincha y Pasa, así como los poblados ubicados a lo largo de esta red vial, considerando necesario obtener información respecto a vivienda, servicios básicos, fuentes de ingresos, densidad poblacional, extensión territorial, crecimiento de la población, ocupación y evolución de los establecimientos económicos.

\section{Marco conceptual}

Hoy en día, la infraestructura vial se ha convertido en un factor esencial en la vida cotidiana de las personas. Respecto a ello, el medio de transporte a utilizar por las personas depende en gran medida de la localidad donde estos residen, ya que algunas zonas, como las suburbanas, cuentan con infraestructura vial en un estado más precario que otras, lo que dificulta la movilidad. El Ministerio de Transporte de Obras Públicas (2013), en su normativa vial NEVI12-MTOP, señala que las afectaciones en la red vial inciden de forma directa y negativa al proceso de desarrollo económico y productivo del Ecuador. 
Los estudios de impacto económico hacen referencia al proceso social que busca generar y distribuir la riqueza (Rodrick, 2003), el cual está determinado por el efecto geográfico, es decir, la localización, lo cual influye en el agrupamiento espacial de las actividades económicas y la correspondiente optimización de los recursos, y que tiene relación directa con el acceso a la movilización vial que faciliten la circulación de los bienes. Por lo que la infraestructura vial es un recurso clave que se juega en el territorio para desarrollo económico, sobre todo si se maneja de manera integral su impacto en el medio ambiente y en la sociedad.

Según el Plan de Desarrollo y Ordenamiento Territorial de Quisapincha (2015), la fuerza productiva y relaciones de producción en la parroquia está dada principalmente por el sector agropecuario y el sector artesanal manufacturero, tal como puede apreciarse en la fabricación de prendas y artículos en cuero en la zona alta de Quisapincha donde se concentra el $70 \%$ de población indígena, la cual depende especialmente de los ingresos generados por la agricultura y crianza de animales (INEC, 2016), de ellos, solo el 14.5\% de la población cubren por completo sus necesidades básicas.

De igual manera, el desarrollo sostenible, que se basa principalmente a los factores medioambientales, económicos y sociales, se centra en proporcionar una calidad aceptable o mejora de vida de las comunidades. En este sentido, los proyectos de infraestructura también deben diseñarse e implementarse conociendo la disponibilidad de recursos y garantizando a las comunidades el acceso a servicios básicos como el alcantarillado, energía eléctrica, recolección de desechos y alumbrado público. Además, se considera que "las instalaciones de carreteras suelen generar una mayor diversidad de tamaños de actividades económicas y estructuras de propiedad" (Corsi, Windle, y Knemeyer, 1999:24).

El Ministerio Coordinador de Producción, Empleo y Competitividad detalla que en el país se ha invertido 6.450 millones de dólares entre el periodo 2007 - 2013 en la construcción y potenciación de alrededor de 9.500 kilómetros de infraestructura vial, lo que ha contribuido al desarrollo productivo de las regiones.

La valoración de los rendimientos o desempeño de las inversiones realizadas en trabajos de infraestructuras están medidos por la variación en las condiciones socioeconómicas que experimenta la población entre dos momentos: antes de la obra y después de la obra. A su vez, los proyectos de construcción de red vial están en concordancia con el objetivo 3 del Plan Nacional del Buen Vivir, el cual busca mejorar la calidad de vida de la población, mediante la reducción de la pobreza, la disminución de las brechas de desigualdad en el incremento de actividades que ayuden al desarrollo económico de la sociedad.

La calidad de vida de las personas hace referencia a aquel conjunto de indicadores que reflejan las condiciones bajo las cuales desarrollan su vida respecto al acceso a la salud, educación y movilización (Arechavala y Trapero, 2009). En el caso del uso de la red vial, Hartmann y Ling (2016) detallan que es frecuente el uso de encuestas de satisfacción a los usuarios de las vías principales y secundarias, tanto a nivel nacional como regional, con el objetivo de obtener información sobre las deficiencias en la red de carreteras y así medir el nivel de satisfacción. 
Y es que las buenas condiciones del espacio físico, rural y urbano contribuyen a generar hábitats saludables mediante el surgimiento de relaciones sociales entre los grupos debido a la disminución de barreras geográficas (Segovia y Jordán Fuchs, 2005). Según estos autores, cuando se realiza una mejora en la red vial en zonas urbanas suele presentarse un efecto domino que conlleva al mejoramiento de aspectos relacionados con el espacio público. Un ejemplo, es la rehabilitación de la vía Ambato-Quisapincha, la cual suscitó que en 1992 se llevarán a cabo la realización de obras para un mejor aprovisionamiento de agua entubada; situación que también se vio motivada por la separación de las Juntas de Agua Potable de la Empresa Municipal de Agua Potable y Alcantarillado Ambato (EMAPA), mejorando la calidad de vida de los pobladores cercanos a la vía.

En el 2000, y posterior al adoquinamiento de las vías urbanas, se inició el alcantarillado para la parroquia Quisapincha, el mismo que ha ido avanzando conforme a obras de infraestructura vial. Además, en el 2005 se da el Proyecto KNH para el desarrollo de la Educación y Salud en Quisapincha, lo cual marcó un referente en el nivel educativo y de salud para la población, proyecto que se vio completado de manera exitosa con la inauguración de la Unidad Educativa del Milenio Quisapincha con infraestructura y equipamiento de punta en el año 2013. No obstante, los desarrollos de mejoras en la infraestructura vial deben respetar las normativas en temas de conservación de recursos naturales; en este sentido, en el 2008 se desarrolla el Plan de Manejo de Páramos con las comunidades del Pueblo Kisapincha cuyo objetivo es guardar y proteger las fuentes de agua (GADM Ambato, 2015).

Asimismo, entre los proyectos más emblemáticos en el ámbito vial, en la provincia de Tungurahua, podemos citar (SENPLADES, 2014): la rehabilitación y mantenimiento de la vía Pelileo-Baños-Puyo, la cual facilita la conectividad entre la zona Sierra y la Amazonía; la ampliación y reconstrucción de la vía Ambato-Pelileo, siendo ésta la red vial que conecta los cantones Ambato y Pelileo, brindando un fuerte impulso a las zonas productivas, artesanales y turísticas; la reparación de las carreteras afectadas por el volcán Tungurahua; la estabilización de taludes del paso lateral Ambato; la rehabilitación de la Vía Ambato-Pasa-Corazón y la construcción de la carretera Cahuají - Pillate- Cotaló.

Las obras públicas antes mencionadas no sólo suponen una mejora en las condiciones de tránsito de la población respecto a fluidez del tránsito vehícular y seguridad vial, sino que también facilita el flujo de mercancías, la conectividad con otras regiones y la circulación de turistas nacionales y extranjeros.

La parroquia Quisapincha, en su Plan de Desarrollo y Ordenamiento Territorial (2015), manifiesta mediante la Agenda Regulatoria que uno de sus objetivos estratégicos a alcanzar en el componente "movilidad" está el "mejorar y ampliar la cobertura de las redes de conectividad, para mantener una población informada y relacionada en su convivir diario", ya que conforme los habitantes viven más lejos de los centros urbanos, más insatisfechas son sus necesidades básicas, la asistencia escolar, la calidad de la vivienda, el acceso a servicios públicos, etc. (Polanco, 2011). Este propósito se vio plasmado en las políticas públicas locales del Gobierno Autónomo de Quisapincha que impulsaban el mejoramiento y mantenimiento de las vías intercomunitarias entre la parroquia y las demás poblaciones aledañas. 


\section{Metodología}

La presente investigación está dirigida al estudio estadístico de percepción de la red vial intercomunitario Quisapincha-Pasa que permitan exponer la situación real de los habitantes de las parroquias y pobladores a lo largo de la vía. El periodo objeto de estudio se centra en el año 2013y 2014, con unas observaciones y actualización de información en los años 2016 y 2017.

Se realiza un análisis cualitativo y cuantitativo, apoyado en fuentes primarias, secundarias, así como del análisis de estadísticas de los datos obtenidos. Investigación de campo, cuyos datos se recabaron de forma directa en el lugar donde se desarrolló la construcción de la vía Quisapincha- Pasa. Para la elaboración del estudio se tomó como referencia los cuestionarios desarrollados por el Instituto Nacional de Estadísticas y Censos (INEC), tanto el Censo de Población y Vivienda efectuado en 2010 como la Encuesta de Condiciones de Vida correspondiente al periodo 2013-2014.

En la investigación se establecieron como elementos de estudio la densidad poblacional, la extensión territorial, el crecimiento de la población, la ocupación de los habitantes y la evolución de los establecimientos económicos. Además se definieron dos dimensiones: dimensión uno, Nivel de Satisfacción y Nivel de Insatisfacción; dimensión dos, Nivel Ni Satisfecho y Nivel Ni Insatisfecho.

La determinación de la población a analizar se determinó a partir de la aplicación de la fórmula de población finita al tener conocimiento de que existen 1124 Jefes de Familia de las parroquias de Quisapincha y Pasa. Una vez aplicada la fórmula se obtuvo un tamaño de la muestra de 401 personas, de los cuales 214 personas son residentes en la parroquia Quisapincha, ubicados en las comunidades de Galpón, Tondolique, Illahua Chico; mientras que los 187 moradores restantes pertenecen a la parroquia Pasa, asentados en las comunidades de Punguloma, Pucaucho y Mogato.

\section{Resultados}

La obra realizada en la carretera intercomunitaria Quisapincha-Pasa ha dado lugar a mejoras que se evidencian en los tipos de vivienda de los pobladores. Como se aprecia en la Tabla 1, el incremento de viviendas tipo Casa/Villa se incrementaron en 8.3 puntos porcentuales entre el año 2010 y el 2013. Asimismo, los datos evidencian una disminución de viviendas tipo Choza en 3.5 puntos porcentuales.

Tabla 1. Comparación del tipo de vivienda, (a) 2010, (b) 2013 


\begin{tabular}{|c|c|c|c|c|c|c|c|}
\hline \multicolumn{4}{|c|}{ (a) } & \multicolumn{4}{|c|}{ (b) } \\
\hline & & Frecuencia & Porcentaje & & & Frecuencia & Porcentaje \\
\hline \multirow{9}{*}{ Válidos } & Casa/villa & 260 & 64,8 & \multirow{9}{*}{ Válidos } & Casa/villa & 293 & 73,1 \\
\hline & Departamento & 6 & 1,5 & & Departamento & 8 & 2,0 \\
\hline & Cuarto & 18 & 4,5 & & Cuarto & 11 & 2,7 \\
\hline & Mediagua & 67 & 16,7 & & Mediagua & 61 & 15,2 \\
\hline & Rancho & 1 & ,2 & & Rancho & 1 &, 2 \\
\hline & Covacha & 6 & 1,5 & & Covacha & 5 & 1,2 \\
\hline & Choza & 31 & 7,7 & & Choza & 17 & 4,2 \\
\hline & Otra & 7 & 1,7 & & Otra & 3 &, 7 \\
\hline & Total & 396 & 98,8 & & Total & 399 & 99,5 \\
\hline Perdidos & Sistema & 5 & 1,2 & Perdidos & Sistema & 2 &, 5 \\
\hline \multicolumn{2}{|l|}{ Total } & 401 & 100,0 & \multicolumn{2}{|l|}{ Total } & 401 & 100,0 \\
\hline
\end{tabular}

Fuente: Base de datos de la Investigación (2013-2014)

La Tabla 2 detalla las vías de acceso utilizadas por los moradores para movilizarse. Se evidencia un incremento significativo en las calles pavimentadas; a la vez, que disminuyen las calles empedradas y, más ligeramente, las calles de tierra.

Tabla 2. Comparación de la vía de acceso, (a) 2010, (b) 2013

\begin{tabular}{|c|c|c|c|}
\hline & & Frecuencia & Porcentaje \\
\hline \multirow{6}{*}{ Válidos } & Calle pavimentada & 40 & 10,0 \\
\hline & Calle empedrada & 185 & 46,1 \\
\hline & Calle tierra & 140 & 34,9 \\
\hline & Camino, sendero & 14 & 3,5 \\
\hline & Otro & 1 & ,2 \\
\hline & Total & 380 & 94,8 \\
\hline Perdidos & Sistema & 21 & 5,2 \\
\hline \multicolumn{2}{|l|}{ Total } & 401 & 100,0 \\
\hline
\end{tabular}

\begin{tabular}{|c|c|c|c|}
\hline & & Frecuencia & Porcentaje \\
\hline \multirow{5}{*}{ Válidos } & Calle pavimentada & 250 & 62,3 \\
\hline & Calle empedrada & 27 & 6,7 \\
\hline & Calle tierra & 96 & 23,9 \\
\hline & Camino, sendero & 8 & 2,0 \\
\hline & Total & 381 & 95,0 \\
\hline Perdidos & Sistema & 20 & 5,0 \\
\hline \multicolumn{2}{|l|}{ Total } & 401 & 100,0 \\
\hline
\end{tabular}

Fuente: Base de datos de la Investigación (2013-2014)

El estudio evidenció que la propiedad de las viviendas es mayoritariamente propiedad de los moradores, tal como muestra la Tabla 3; mientras que las viviendas en alquiler y prestadas disminuyen ligeramente.

Tabla 3. Comparación - Propiedad de la vivienda, (a) 2010, (b) 2013

\begin{tabular}{|c|c|c|c|}
\hline & & Frecuencia & Porcentaje \\
\hline \multirow{6}{*}{ Válidos } & $\begin{array}{l}\text { propia y totalmente } \\
\text { pagada }\end{array}$ & 301 & 75,1 \\
\hline & $\begin{array}{l}\text { propia y la está } \\
\text { pagando }\end{array}$ & 24 & 6,0 \\
\hline & propia & 50 & 12,5 \\
\hline & $\begin{array}{l}\text { Prestada o cedida } \\
\text { no paga }\end{array}$ & 13 & 3,2 \\
\hline & arrendada & 6 & 1,5 \\
\hline & Total & 394 & 98,3 \\
\hline Perdidos & Sistema & 7 & 1,7 \\
\hline \multicolumn{2}{|l|}{ Total } & 401 & 100,0 \\
\hline
\end{tabular}

\begin{tabular}{|c|c|c|c|}
\hline \multicolumn{4}{|c|}{ (b) } \\
\hline & & Frecuencia & Porcentaje \\
\hline \multirow{6}{*}{ Válidos } & $\begin{array}{l}\text { propia y totalmente } \\
\text { pagada }\end{array}$ & 312 & 77,8 \\
\hline & $\begin{array}{l}\text { propia y la está } \\
\text { pagando }\end{array}$ & 27 & 6,7 \\
\hline & propia & 48 & 12,0 \\
\hline & $\begin{array}{l}\text { Prestada o cedida } \\
\text { no paga }\end{array}$ & 7 & 1,7 \\
\hline & arrendada & 2 &, 5 \\
\hline & Total & 396 & 98,8 \\
\hline Perdidos & Sistema & 5 & 1,2 \\
\hline \multicolumn{2}{|l|}{ Total } & 401 & 100,0 \\
\hline
\end{tabular}

Fuente: Base de datos de la Investigación (2013-2014) 
Una de las mejoras más evidentes es el uso del bus como medio de transporte que comunica a los moradores con las distintas localidades de la provincia. En el año 2010 el $16 \%$ de la población se trasladaba en bus, mientras que en 2013 el uso del bus como medio de transporte se incrementa a un $46.9 \%$ (Tabla 4); a su vez, los traslados en camioneta y furgoneta disminuyen.

Tabla 4. Comparación medio de transporte, (a) 2010, (b) 2013

(s)

\begin{tabular}{|l|l|c|c|}
\hline \multicolumn{2}{|c|}{} & Frecuencia & Porcentaje \\
\hline \multirow{4}{*}{} & bus & 64 & 16,0 \\
\cline { 2 - 4 } & camioneta & 298 & 74,3 \\
\cline { 2 - 4 } & furgoneta & 3 &, 7 \\
\cline { 2 - 4 } Válidos & camión & 8 & 2,0 \\
\cline { 2 - 4 } & vehículo particular & 9 & 2,2 \\
\cline { 2 - 4 } & atros & 12 & 3,0 \\
\cline { 2 - 4 } & Total & 394 & 98,3 \\
\hline \multirow{4}{*}{ Perdidos } & Sistema & 7 & 1,7 \\
\hline \multirow{2}{*}{ Total } & & 401 & 100,0 \\
\hline
\end{tabular}

\begin{tabular}{|l|l|c|c|}
\hline \multicolumn{2}{|c|}{} & Frecuencia & Porcentaje \\
\hline \multirow{4}{*}{} & bus & 188 & 46,9 \\
\cline { 2 - 4 } & camioneta & 187 & 46,6 \\
\cline { 2 - 4 } & furgoneta & 2 &, 5 \\
\cline { 2 - 4 } & camión & 1 &, 2 \\
\cline { 2 - 4 } & vehículo particular & 17 & 4,2 \\
\cline { 2 - 4 } & Total & 395 & 98,5 \\
\hline Perdidos & Sisterna & 6 & 1,5 \\
\hline \multirow{2}{*}{ Total } & & 401 & 100,0 \\
\hline
\end{tabular}

Fuente: Base de datos de la Investigación (2013-2014)

Respecto a la calidad de vida de los moradores de la red vial intercomunitaria Quisapincha-Pasa se aprecia en un incremento de 6.2 entre los años 2010 y 2013 en el uso de la red pública como acceso al recurso agua (Tabla 5). En el mismo periodo se observa una disminución en el uso de agua procedente de otras fuentes, tales como pozo, río/vertientes y carro repartidor.

Tabla 5. Comparación de la procedencia del agua, (a) 2010 y (b) 2013

\begin{tabular}{|l|l|c|c|}
\hline \multicolumn{4}{|c}{ (a) } \\
\hline \multirow{4}{*}{} & Red publica & Frecuencia & Porcentaje \\
\cline { 2 - 4 } & Pozo & 78 & 19,5 \\
\cline { 2 - 4 } & Rio, vertiente, & 24 & 6,0 \\
\hline \multirow{4}{*}{ Válidos } & acequia o canal & 278 & 69,3 \\
\cline { 2 - 4 } & Carro repartidor & 2 &, 5 \\
\cline { 2 - 4 } & Junta parroquial & 17 & 4,2 \\
\cline { 2 - 4 } & Otro & 1 &, 2 \\
\cline { 2 - 4 } & Total & 400 & 99,8 \\
\hline \multirow{2}{*}{ Perdidos } & Sistema & 1 &, 2 \\
\hline \multirow{2}{*}{ Total } & & 401 & 100,0 \\
\hline
\end{tabular}

\begin{tabular}{|c|c|c|c|}
\hline \multicolumn{4}{|c|}{ (b) } \\
\hline & & Frecuencia & Porcentaje \\
\hline \multirow{7}{*}{ Válidos } & Red publica & 103 & 25,7 \\
\hline & Pozo & 21 & 5,2 \\
\hline & $\begin{array}{l}\text { Rio, vertiente, } \\
\text { acequia o canal }\end{array}$ & 252 & 62,8 \\
\hline & Carro repartidor & 1 & $r^{2}$ \\
\hline & Junta parroquial & 19 & 4,7 \\
\hline & Otro & 1 & 2 \\
\hline & Total & 397 & 99,0 \\
\hline Perdidos & Sistema & 4 & 1,0 \\
\hline Total & & 401 & 100,0 \\
\hline
\end{tabular}

Fuente: Base de datos de la Investigación (2013-2014)

La Tabla 6 muestra un incremento significativo en el uso de la red pública de alcantarillado, pasando de 27,2\% al 50,6\%, entre los años 2010 y 2013, así como una disminución en el porcentaje de pobladores quienes no tenían en sus viviendas el uso de letrinas.

Tabla 6. Comparación del acceso al servicio higiénico (a) 2010 y (b) 2013 


\begin{tabular}{|c|c|c|c|}
\hline & & Frecuencia & Porcentaje \\
\hline \multirow{7}{*}{ Válidos } & $\begin{array}{l}\text { red pública } \\
\text { alcantarillado }\end{array}$ & 109 & 27,2 \\
\hline & pozo séptico & 55 & 13,7 \\
\hline & pozo ciego & 193 & 48,1 \\
\hline & $\begin{array}{l}\text { descarga directa } \\
\text { quebrada }\end{array}$ & 8 & 2,0 \\
\hline & letrina & 10 & 2,5 \\
\hline & no tiene & 21 & 5,2 \\
\hline & Total & 396 & 98,8 \\
\hline Perdidos & Sistema & 5 & 1,2 \\
\hline \multicolumn{2}{|l|}{ Total } & 401 & 100,0 \\
\hline
\end{tabular}

\begin{tabular}{|c|c|c|c|}
\hline & & Frecuencia & Porcentaje \\
\hline \multirow{7}{*}{ Válidos } & $\begin{array}{l}\text { red pública } \\
\text { alcantarillado }\end{array}$ & 203 & 50,6 \\
\hline & pozo séptico & 35 & 8,7 \\
\hline & pozo ciego & 131 & 32,7 \\
\hline & $\begin{array}{l}\text { descarga directa } \\
\text { quebrada }\end{array}$ & 5 & 1,2 \\
\hline & letrina & 8 & 2,0 \\
\hline & no tiene & 14 & 3,5 \\
\hline & Total & 396 & 98,8 \\
\hline Perdidos & Sisterna & 5 & 1,2 \\
\hline \multicolumn{2}{|l|}{ Total } & 401 & 100,0 \\
\hline
\end{tabular}

Fuente: Base de datos de la Investigación (2013-2014)

Otro de los servicios en donde se aprecian mejorías es en el alumbrado público (Tabla 7), donde se observa que en el año 2010 apenas el 16,7\% de los pobladores disponían de este servicio; afortunadamente para estos habitantes, en el 2013, éste servicio ha llegado a su alcance, aumentado el porcentaje a 53,6\%.

Tabla 7.Comparación alumbrado público, (a) 2010, (b) 2013

\begin{tabular}{|c|c|c|c|c|c|c|c|}
\hline \multicolumn{4}{|c|}{ (a) } & \multicolumn{4}{|c|}{ (b) } \\
\hline & & Frecuencia & Porcentaje & & & Frecuencia & Porcentaje \\
\hline \multirow{3}{*}{ Válidos } & si & 67 & 16,7 & \multirow{3}{*}{ Válidos } & si & 215 & 53,6 \\
\hline & no & 331 & 82,5 & & no & 182 & 45,4 \\
\hline & Total & 398 & 99,3 & & Total & 397 & 99,0 \\
\hline Perdidos & Sistema & 3 &, 7 & Perdidos & Sistema & 4 & 1,0 \\
\hline \multicolumn{2}{|l|}{ Total } & 401 & 100,0 & \multicolumn{2}{|l|}{ Total } & 401 & 100,0 \\
\hline
\end{tabular}

Fuente: Base de datos de la Investigación (2013-2014)

En la Tabla 8 se aprecia que el jefe de familia se dedica principalmente a la agricultura, actividad que ha decrecido levemente en el transcurso del tiempo, pasando del 63,6\% al 62,6\%; a la vez que, el sector servicios ha ganado terreno. Esto se debe a que al incorporar un mejoramiento en la red vial se produce una diversificación de actividades y un cambio en los patrones de consumo de los pobladores.

Tabla 8. Comparación respecto a la dedicación del jefe de familia, (a) 2010, (b) 2013

\begin{tabular}{|c|c|c|c|}
\hline & & Frecuencia & Porcentaje \\
\hline \multirow{5}{*}{ Válidos } & "agricultura" & 255 & 63,6 \\
\hline & "comercio" & 24 & 6,0 \\
\hline & "industria" & 19 & 4,7 \\
\hline & "servicios" & 83 & 20,7 \\
\hline & Total & 381 & 95,0 \\
\hline Perdidos & Sistema & 20 & 5,0 \\
\hline \multicolumn{2}{|l|}{ Total } & 401 & 100,0 \\
\hline
\end{tabular}

\begin{tabular}{|l|l|c|c|}
\hline \multicolumn{2}{|c|}{} & (b) \\
\hline \multirow{4}{*}{ Válidos } & "agricultura" & 251 & 62,6 \\
\cline { 2 - 4 } & "comercio" & 22 & 5,5 \\
\cline { 2 - 4 } & "industria" & 19 & 4,7 \\
\cline { 2 - 4 } & "servicios" & 88 & 21,9 \\
\cline { 2 - 4 } & Total & 380 & 94,8 \\
\hline \multirow{2}{*}{ Perdidos } & Sistema & 21 & 5,2 \\
\hline Total & & 401 & 100,0 \\
\hline
\end{tabular}

Fuente: Base de datos de la Investigación (2013-2014) 
Otra información que consideramos relevante conocer son los ingresos familiares, así como su procedencia, es decir, los ingresos por parte del jefe de familia y el de los otros miembros de la unidad familiar.

En la Tabla 9 se observa que el importe de ingresos promedio por unidad familiar es de $\$ 369,86$ para el año 2010 y $\$ 464,01$ para 2013; tomando en cuenta, que en el 2010 el salario básico unificado (SBU) se estableció en \$240,00 y la canasta familiar básica en \$544,71. En el año 2013, el salario básico unificado incremento un 32,5 \% en relación al 2010, alcanzando el importe de $\$ 318,00$; de igual manera, el costo de la canasta familiar básica para el 2013 presentó un aumento de $13,98 \%$ estableciéndose en $\$ 620,86$.

Como puede apreciarse, existe una amplia variabilidad, es decir, la existencia de rentas muy bajas y rentas muy altas de manera dispersa, así como diferencias entre los ingresos promedios y el costo de la canasta familiar básica.

Tabla 9. Comparación de ingresos familiares 2010-2013

\begin{tabular}{|c|c|c|c|c|c|c|}
\hline \multicolumn{2}{|c|}{} & Media & Des. Std. & Rango & Mínimo & Máximo \\
\hline \multirow{2}{*}{ Jefe familia } & Año 2010 & 256.31 & 382.82 & 6000 & 0 & 6000 \\
\cline { 2 - 7 } & Año 2013 & 308.02 & 415.88 & 6000 & 0 & 6000 \\
\hline \multirow{2}{*}{$\begin{array}{c}\text { Otros miembros } \\
\text { familia }\end{array}$} & Año 2010 & 113.55 & 222.39 & 2500 & 0 & 2500 \\
\cline { 2 - 8 } & Año 2013 & 155.99 & 329.46 & 3540 & 0 & 3540 \\
\hline
\end{tabular}

Fuente: Base de datos de la Investigación (2013-2014)

El análisis del nivel socioeconómico de los pobladores de la vía intercomunitaria Quisapincha - Pasa muestra que no se han detectado variaciones significativas a lo largo del tiempo en ninguna de las categorías. Así, en el 2010 predomina la clase baja con un 50.6\%, cuya cantidad descendió hasta el $46.9 \%$ en el 2013. Se aprecia un leve aumento en la categoría de nivel medio y alto en $3,0 \%$ y $1,3 \%$ respectivamente.

Tabla 10. Comparación del nivel socio económico, (a) 2010 y (b) 2013

\begin{tabular}{|l|l|c|c|}
\hline \multicolumn{2}{|c|}{} & (a) \\
\hline \multirow{4}{*}{ Válidos } & bajo & 203 & 50,6 \\
\cline { 2 - 4 } & medio & 166 & 41,4 \\
\cline { 2 - 4 } & alto & 19 & 4,7 \\
\cline { 2 - 4 } & Total & 388 & 96,8 \\
\hline \multirow{2}{*}{ Perdidos } & Sistema & 13 & 3,2 \\
\hline \multirow{2}{*}{ Total } & & 401 & 100,0 \\
\hline
\end{tabular}

\begin{tabular}{|l|l|c|c|}
\hline \multicolumn{2}{|c}{ (b) } & Frecuencia & Porcentaje \\
\hline \multirow{4}{*}{ Válidos } & bajo & 188 & 46,9 \\
\cline { 2 - 4 } & medio & 178 & 44,4 \\
\cline { 2 - 4 } & alto & 24 & 6,0 \\
\cline { 2 - 4 } & Total & 390 & 97,3 \\
\hline \multirow{2}{*}{ Perdidos } & Sistema & 11 & 2,7 \\
\hline \multirow{2}{*}{ Total } & & 401 & 100,0 \\
\hline
\end{tabular}

Fuente: Base de datos de la Investigación (2013-2014) 
Las obras en la red vial animan a los pobladores a adquirir vehículos, es decir, un crecimiento en el parque automotor de la zona. En este contexto, se evidencia que entre el año 2010 y 2013 incrementó un 3,0\% el porcentaje de pobladores dueños de un vehículo.

Tabla 12. Posee vehículo propio, (a) 2010, (b) 2013

(a)

\begin{tabular}{|l|l|c|c|}
\hline \multicolumn{2}{|c|}{} & Frecuencia & Porcentaje \\
\hline \multirow{3}{*}{ Válidos } & si & 30 & 7,5 \\
\cline { 2 - 4 } & no & 352 & 87,8 \\
\cline { 2 - 4 } & Total & 382 & 95,3 \\
\hline \multirow{2}{*}{ Perdidos } & Sistema & 19 & 4,7 \\
\hline \multirow{2}{*}{ Total } & & 401 & 100,0 \\
\hline
\end{tabular}

(b)

\begin{tabular}{|l|l|c|c|}
\hline \multicolumn{2}{|c|}{} & Frecuencia & Porcentaje \\
\hline \multirow{3}{*}{ Válidos } & si & 42 & 10,5 \\
\cline { 2 - 4 } & no & 338 & 84,3 \\
\cline { 2 - 4 } & Total & 380 & 94,8 \\
\hline \multirow{2}{*}{ Perdidos } & Sistema & 21 & 5,2 \\
\hline \multirow{2}{*}{ Total } & & 401 & 100,0 \\
\hline
\end{tabular}

Fuente: Base de datos de la Investigación (2013-2014)

\section{Análisis Multidimensional}

Para el análisis del Estado de Satisfacción que ha proporcionado las obras en la vía intercomunitaria Quisapincha-Pasa se ha utilizado el Análisis de Correspondencias Múltiples (ACM). Para el cálculo del ACM se han definido las variables Parroquias, Sector y las correspondientes al Nivel de Satisfacción. Las variables Parroquia y Sector son tomadas como variables suplementarias, es decir, aquellas proyectadas sobre el espacio formado por las variables de satisfacción.

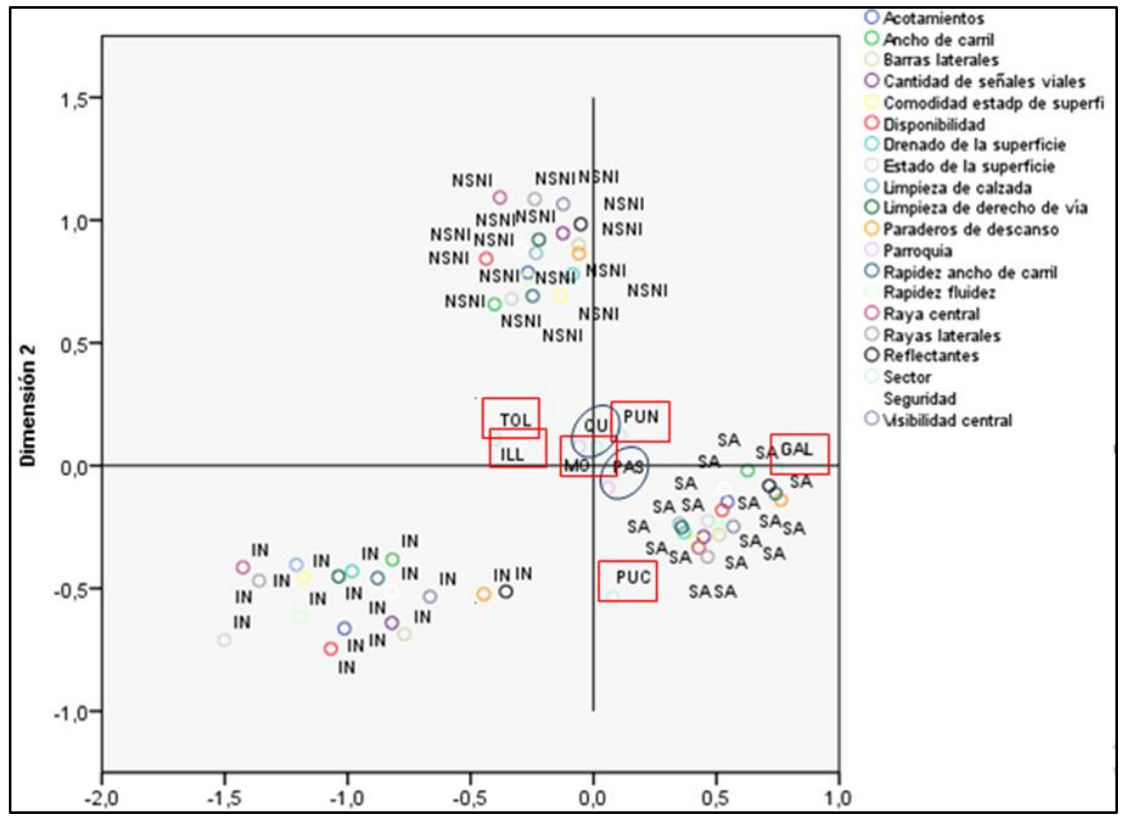

Figura 1. Plano factorial de las modalidades de las variables de Nivel de Satisfacción de la carretera, Parroquia y Sector.

Fuente: Encuesta realizada a los jefes de familia del sector (2014) 
De los resultados que se muestran en la Figura 1 se pueden obtener las siguientes conclusiones:

- El 1er. eje factorial se caracteriza por discriminar entre nivel de satisfacción y nivel de insatisfacción.

- El 2do. eje factorial se caracteriza por representar a los pobladores ni satisfecho ni insatisfecho

- La parroquia Pasa (PAS) cae en la región Satisfacción.

- La parroquia Quisapincha (QUI) cae en la región de indecisión, ni satisfecho ni insatisfecho.

- Los sectores Galpón y Pul están completamente satisfechos respecto a la calidad de la red vial.

- Los sectores Tonlolique (TOL) e Illagua (ILL) están insatisfechos respecto a la calidad de la vía intercomunitaria.

- Los sectores Punguloma (PUN) y Mogato (MO) no indican una decisión clara de satisfacción ni de insatisfacción respecto a la calidad de la vía.

- Debido a que ninguna parroquia y ningún sector caen en la zona de insatisfacción se puede concluir que la construcción de la vía intercomunitaria Quisapincha-Pasa si cuenta con el apoyo de los pobladores involucrados.

\section{Índice de mejora en la construcción de la carretera}

Para la elaboración de un Índice, que mida los beneficios que puede producir en los pobladores de una red vial la construcción de una carretera, se ha utilizado el denominado Análisis de Componentes Principales (Santos y Gomes, 2015). Esta técnica estadística permite realizar una síntesis de la información recogida en la investigación.

Los resultados de este análisis se presentan en la Tabla X, donde se detallan los autovalores; mientras que en la Tabla X se muestran los aportes que hacen cada una de las variables a los respectivos componentes. Se debe tomar en consideración que se toma como base el año 2010. Tomando como umbral de corte el valor de 0,5 se obtienen 7 componentes, los mismos que están representados en la Tabla X.

Tabla 13. Componentes representativos del estudio

\begin{tabular}{cl}
\hline Nombre componente & \multicolumn{1}{c}{ Variables } \\
\hline C1 & EstadoTecho2010 \\
& EstadoParedes2010 \\
& MaterialPiso2010 \\
& EstadoPiso2010 \\
& CombustibleCocina2010 \\
& VehiculoPropio2010 \\
& Combustiblevehiculo2010 \\
& EstadoTecho2010 \\
& EstadoParedes2010 \\
& PagoMatricula2010 \\
& TipoVivieneda2010 \\
& DisponeMedidor2010 \\
& AccidentesTransito2010 \\
\hline
\end{tabular}




\begin{tabular}{ll}
\hline C5 & Internet2010 \\
C6 & EstudiaFueraParroquia2010 \\
C7 & TvCable2010 \\
& NivelSocioeconomico2010 \\
\hline
\end{tabular}

Elaboración: Las autoras.

Mediante la aplicación de la ecuación (1) se obtiene los siguientes valores:

$$
M=\frac{1}{7} \sum_{i=1}^{7} C_{i}
$$

Tras el análisis de los siete componentes, los resultados obtenidos son:

- $M_{2010}=1.78$

- $M_{2013}=1.55$

Como se puede observar, el valor correspondiente al año 2013 muestra una percepción de beneficios producidas por la construcción de la red vial intercomunitaria Quisapincha-Pasa, concluyendo también que se produjo una mejora en la calidad de vida de $12.49 \%$.

Tabla 14. Medidas de discriminación de la satisfacción poblacional

\begin{tabular}{|c|c|c|c|}
\hline \multirow[t]{2}{*}{ Detalles } & \multicolumn{2}{|c|}{ Dimension } & \multirow[t]{2}{*}{ Media } \\
\hline & 1 & 2 & \\
\hline Ancho de carril & ,424 &, 123 & ,273 \\
\hline Acotamientos & ,383 & ,263 & ,323 \\
\hline Estado de la superficie & ,403 & ,223 & ,313 \\
\hline Drenado de la superficie & ,244 &, 242 & ,243 \\
\hline Cantidad de señales viales ${ }^{a}$ & 107 &, 030 & 069 \\
\hline Visibilidad central & ,273 & ,393 & ,333 \\
\hline Raya central & ,394 &, 382 & ,388 \\
\hline Rayas laterales & ,410 & ,426 & ,418 \\
\hline Barras laterales & ,268 & ,406 & ,337 \\
\hline Reflectantes & ,203 &, 382 & ,292 \\
\hline $\begin{array}{l}\text { Comodidad estado de } \\
\text { superficie }\end{array}$ & ,301 & ,234 & ,268 \\
\hline Paraderos de descanso & ,247 &, 333 & ,290 \\
\hline Rapidez ancho de carril & ,473 &, 196 & ,335 \\
\hline Rapidez fluidez & ,381 & ,266 & ,323 \\
\hline Disponibilidad & ,381 &, 300 & ,340 \\
\hline Limpieza de calzada & ,305 &, 242 & ,274 \\
\hline Limpieza de derecho de vía & ,267 &, 280 & ,274 \\
\hline Parroquia $^{a}$ & ,001 & ,003 & ,002 \\
\hline Basura 2010 & ,011 & 037 & ,024 \\
\hline Basura 2013 ${ }^{a}$ &, 021 & ,028 &, 024 \\
\hline $\begin{array}{l}\text { Total activo } \\
\text { a. Variable suplementaria. }\end{array}$ & 5,946 & 5,318 & 5,632 \\
\hline
\end{tabular}

Fuente: Encuesta realizada a los jefes de familia del sector (2014)

Tabla 15. Análisis estadístico

\begin{tabular}{lll}
\hline Dimensión & Alfa de Cronbach & Varianza explicada \\
\hline
\end{tabular}




\begin{tabular}{cccc}
\hline & & Total (Autovalores) & Inercia \\
$\mathbf{1}$ &, 881 & 5,946 &, 330 \\
$\mathbf{2}$ &, 860 & 5,318 &, 295 \\
Total & & 11,265 &, 626 \\
Media &, $871^{\text {a }}$ & 5,632 &, 313 \\
\hline Fuente: Encuesta realizada a los jefes de familia del sector (2014)
\end{tabular}

Asimismo, se evidencia la fiabilidad de los datos, medidos con el Alfa de Cronbach: concretamente, la Dimensión 1 presenta un coeficiente bueno $(0,881)$ y la Dimensión 2 también un coeficiente bueno, aunque levemente inferior $(0,860)$.

\section{Discusión}

La construcción de la red vial intercomunitaria Quisapincha-Pasa ha contribuido a mejorar aspectos fundamentales como la accesibilidad, movilidad y acceso a servicios básicos por parte de los pobladores; de igual manera, se ha podido presenciar un impacto económico positivo ya que al implementar mejoras en las vías de comunicación se facilita el transporte de bienes y personas.

Se verificó que, al medir el nivel de satisfacción a través del Análisis de Componentes Principales, la construcción vial sí se encuentra respaldada por los pobladores aledaños a la misma. Sin embargo, se desconoce el número total de trabajadores residentes en la zona, quienes participaron en la construcción de la red vial intercomunitaria Quisapincha-Pasa, lo que se traduce en el principal limitante del estudio.

\section{Conclusiones}

A pesar de las numerosas obras realizadas en las vías principales e intercomunitarias a nivel nacional, todavía persisten algunas zonas poblacionales con problemas de movilidad debido a la ausencia de camino adecuados. Los indicadores de calidad de vida considerados en la investigación proporcionaron información sobre la existencia de una mejora en el tipo de vivienda de los pobladores de la vía comunitaria Quisapincha-Pasa; asimismo, de evidencia un crecimiento en el acceso a distintos medios de transporte, principalmente la utilización de bus, y de servicios básicos, tales como: agua potable, alcantarillado y alumbrado público.

El incremento en el número de calles pavimentadas ha contribuido a que los pobladores tengan mayor accesibilidad a otros recursos, así como el acercamiento a las urbes más cercanas. Mediante el Análisis de Correspondencias Múltiples se obtuvo que el estado de satisfacción de los pobladores pertenecientes a los sectores que componen la vía Quisapincha-Pasa, dando como resultado al no caer ningún sector en la zona de insatisfacción, que la construcción de la vía intercomunitaria si contó con el apoyo de los pobladores involucrados.

De igual manera, el análisis de las dimensiones consideradas en el estudio dio como resultado, tomando como referencia el año 2010, un índice de calidad de vida de $12,49 \%$, esto es que existe una mejora en la calidad de vida de los pobladores. Finalmente, se puede concluir que las obras realizadas en la vía intercomunitaria Quisapincha-Pasa han brindado a los pobladores 
de los distintos sectores la oportunidad de mejorar sus condiciones de vida, así como proporcionar una mejor movilidad de bienes y personas con las demás comunidades.

\section{Bibliografía}

Arechavala, N. S., y Trapero, J. B. P. (2009). La medición de la calidad de vida en Europa, el papel de la información subjetiva. Revista de Estudios de economía aplicada, Vol. 27, No 2, pp373-396.

Corsi, T. M., Windle, R. J., y Knemeyer, A. M. (1999). Evaluating the potential impact of interstate highway rights-of-way commercialization on economic activity at interchanges. Transportation journal, Vol. 39, No 2, pp.16-25.

Gobierno Autónomo Descentralizado Municipal de Ambato - GADM Ambato (2015): Actualización del Plan de Desarrollo y Ordenamiento Territorial.

Instituto Nacional de Estadísticas y Censos - INEC (2010): Censo de Población y Vivienda.

Instituto Nacional de Estadísticas y Censos - INEC (2013): Encuesta de Pobreza por Necesidades Básicas Insatisfechas - NBI.

Hartmann, A. and Ling, F.Y.Y. (2016). Value creation of road infrastructure networks: a structural equation approach. Journal of Traffic and Transportation Engineering, Vol. 3 No. 1, pp. 28 -36 .

Polanco, J. A. (2011). Incidencia del desarrollo socio-económico en la perennidad del páramo. Gestión y Ambiente, Vol. 14, No 1, pp. 117-128.

Rydin, Y., Bleahu, A., Davies, M., Dávila, J.D., Friel, S., De Grandis, G., Groce, N., Hallal, P.C., Hamilton, I., Howden-Chapman, P., (2012). Shaping cities for health: complexity and the planning of urban environments in the 21st century. Lancet No. 379, 2079.

Rodrik, D. (2003). In search of prosperity: Analytic narratives on economic growth. Princeton University Press. United State of America. 481p.

Santos, J. L. D. C., y Gomes, G. M. F. (2015). Estudios para el plan de accesibilidad a territorios de similar identidad: Estado de Bahía. Urbano, Vol. 15, No 25, pp. 57-66.

Secretaría Nacional de Planificación y Desarrollo - SENPLADES (2014). Proyectos emblemáticos en Tungurahua.

Segovia, O., \& Jordán Fuchs, R. (2005). Espacios públicos urbanos, pobreza y construcción social. CEPAL. División de Desarrollo Sostenible y Asentamientos Humanos. Chile. No 122, 52p.

Villar, L., y Ramírez, J. M. (2014). Infraestructura regional y pobreza rural. Fedesarrollo, Centro de Investigación Económica y Social. Working paper No. 61 (2014-2), pp. 1-52. 